\title{
LA MALADIE DE THÉRÈSE MARTIN ET LA GUERISON PAR LA VIERGE MARIE
}

Avant de traiter en théologien de la maladie et de la guérison de Thérèse, dont on trouve le récit au Manuscrit A $27 \mathrm{r}^{\circ}-30 \mathrm{v}^{\circ}$, il est nécessaire d'en rapporter l'opinion des médecins, car l'interprétation spirituelle ne dispense pas de l'interprétation psychologique, «l'une ne rend pas l'autre superflue $»^{1}$. Nous respecterons les divers diagnostics médicaux, si divergents soient-ils, à condition qu'ils n'excluent pas la vision théologique, comme l'a écrit De Meester :

On parle de retard affectif, de névrose, avec le point culminant dans la maladie à l'âge de dix ans, d'obsession névrotique pendant sa crise de scrupules. Laissant ce domaine aux gens de métier, nous n'aurons aucune difficulté à admettre ces troubles psychologiques si vraisemblables chez Thérèse enfant, à condition que le travail purificateur de Dieu exercé au moyen des causes naturelles et la très généreuse, bien que psychologiquement insuffisante, réaction de Thérèse ne soient pas mis en cause. La Sainte en sort encore plus humaine et plus proche de nous ${ }^{2}$.

1 Jean Paul II, Foi et raison, n. 9. Dans son encyclique, Jean Paul II rappelle que deux ordres de connaissance, foi et raison, sont distincts et complémentaires.

2 C. De Meester, Dynamique de la confiance. Genèse et structure de la "voie d'enfance spirituelle » de sainte Thérèse de Lisieux, Paris $1995^{2}$ (éd. révisée), p. 25. H.U. von Balthasar combat les tentatives d'enfermer Thérèse dans des interprétations exclusivement psychologiques : "Qu'était-ce que la religiosité des Buissonnets ? C'est là tout au plus une question de matière et on connaît assez la liberté que prit Thérèse vis-à-vis d'elle pour la subordonner à la propre vocation. Pour le grand artiste, toute matière (même imparfaite) est comme une disposition ultime bienvenue; elle lui est aussi nécessaire que Christian Bach l'a été pour Mozart, ou les contes italiens pour Shakespeare ou la romance espagnole pour Corneille. Mais aucune matière, pour préparée qu'elle soit, ne laissera soupçonner la forme géniale qui doit en sortir. De là vient que des psychologues et des psychanalystes perdent leur temps auprès de Thérèse ; de même les médecins qui se lancent dans des conjectures sur la maladie de sa jeunesse » (H.U. von Balthasar, Actualité de Lisieux, « Nouvelles de l'Institut Catholique de Paris » 2 [nº spécial, mai 1973], p. 107-108). 
Considérons donc la maladie, telle qu'elle a paru aux yeux de ses proches, à ses médecins impuissants à émettre un diagnostic et à ceux d'aujourd'hui.

\section{Psychologie}

Le 2 octobre 1882, Pauline entre au Carmel. Thérèse dépend trop de sa seconde mère pour ne pas ressentir les effets néfastes de cette séparation ${ }^{3}$. Peu après, elle est prise de maux de tête continuels, et de douleurs au côté et au cœur. Ils durent six mois et sont les prodromes d'une maladie dont personne ne se doute. Celle-ci se déclenche subitement à Pâques, le 23 mars 1883, après que l'oncle Guérin a évoqué imprudemment le souvenir de la mère de Thérèse, alors que son père et ses deux sœurs aînées sont à Paris. Elle durera six semaines avec une rémission le jour de la prise d'habit de Pauline, le 6 avril, puis cessera brusquement à la Pentecôte, le 13 mai. Jeanne Guérin se souvient:

Ce mal débuta par un tremblement violent qui fit croire d'abord à une fièvre. Puis se manifesta avec de la dépression, un état de demi hallucination qui lui faisait voir les différents objets ou les attitudes de ceux qui l'entouraient, sous des formes effrayantes. A la période la plus intense, il y eut aussi plusieurs crises motrices pendant lesquelles elle réalisait des mouvements rotatoires de tout le corps, dont elle eût été absolument incapable en état de santé4 .

Avec tout cela, Thérèse assure avoir gardé toute sa lucidité : «je suis sûre de n'avoir pas été privée un seul instant de l'usage de ma raison... .".

Le docteur Notta, appelé auprès de la malade, est déconcerté. Il avoue n’avoir rien compris à la maladie, d'où son diagnostic imprécis : "Maladie très grave et dont jamais une enfant si jeune n'avait été atteinte $»^{6}$.

3 Pour les membres de la famille et son entourage, le lien entre la « disparition » de Pauline et la maladie de Thérèse était évident : «A l'âge de 10 ans, elle fut prise d'une maladie terrible que nous attribuâmes au grand chagrin qu'elle eut du départ de sa sœur Pauline pour le Carmel: je me rappelle fort bien qu'elle en avait un chagrin très profond et que rien ne pouvait la consoler " (Jeanne Guérin, PO, p. 493). «Cette séparation avait été très douloureuse à la Servante de Dieu et elle en avait conçu, je crois, une tristesse qu'elle s'efforçait de comprimer et qui occasionna, sans doute, au moins partiellement, la maladie qui se déclara subitement à cette époque (Marcelline-Anne, bonne des Guérin, devenue sœur Marie-Joseph de la Croix, O.S.B., PO, p. 363).

4 Jeanne Guérin, PA, p. 515. On trouvera les dépositions sur la maladie des témoins oculaires suivants : Jeanne Guérin, PO, p. 493 et PA, p. 515; Marcelline-Anne Husé, bonne des Guérin, devenue sœur Marie-Joseph de la Croix, O.S. B., PO, p. 363-364 et PA, p. 510 ; Léonie, PO, p. 343-344; Marie du Sacré-Cœur, PO, p. 340-341 et PA, p. 227-228; Sœur Geneviève, PO, p. 302-303.

5 A $28 \mathrm{v}^{\circ}$.

6 A $27 \mathrm{v}^{\circ}$ 
Plus d'un demi-siècle après, le professeur Gayral de la faculté de Médecine de Toulouse n'éprouva pas les mêmes difficultés à déterminer la maladie : il s'agit pour lui d'une névrose pithiatique infantile. Dans son étude, qui mérite une attention particulière pour sa compétence et son art de la synthèse, il écrit :

Thérèse présentait un retard affectif consécutif à la régression causée par la perte de sa mère et non compensée par une éducation suffisamment compréhensive. [...] Elle se comporte comme un petit bébé qui réclame sa mère 'Mama, Mama', tyrannise son entourage surtout Marie, qui devait sans cesse rester auprès de son lit. Dans la maladie, elle peut satisfaire ses besoins insatisfaits d'être soignée, dorlotée et irresponsable et ce résultat de la maladie en est le but profond. [...] En définitive elle ne revient à elle-même que réconfortée par la vision de ce qui pour elle est la Mère par excellence?.

Cette opinion est aujourd'hui largement partagée. Signalons toutefois l'existence d'autres hypothèses. Pour le docteur Masson, la maladie serait une tuberculose cérébrale $^{8}$, idée peu soutenable . Quant à l'hystérie, beaucoup de symptômes auraient prévalu en faveur d'un tel diagnostic. Mais le docteur Notta a formellement exclu cette hypothèse : «Qu'on appelle cela du nom que l'on voudra, mais pour moi, ce n'est pas de l'hystérie " ${ }^{10}$, le principal obstacle étant l'âge trop jeune de l'enfant. Isidore Guérin rapporte les propos du médecin : «Si ces symptômes s'étaient produits vers l'âge de 14 ou 15 ans, [le docteur] les aurait peut-être compris, mais chez une enfant de 10 ans ils étaient inexplicables ${ }^{11}$. D'autres médecins, plus tard, y verront pourtant «une maladie hystérique grave $»^{12}$.

Enfin, il serait intéressant de prendre en considération le point de vue du psychiatre polonais Kazimierz Dąbrowski (1902-1980), avec la théorie de la désintégration positive. D'après lui, la névrose ne serait pas un signe de maladie et de régression, mais de croissance psychologique ${ }^{13}$. En effet, le développement de la personnalité ne

7 L.-F. Gayral, Une maladie nerveuse dans l'enfance de Ste Thérèse de Lisieux, "Carmel » 2 (1959), p. $93,85-86,94$.

8 Voir R. Masson, Souffrance des hommes. Un psychiatre interroge Thérèse de Lisieux, Versailles 1997, p. 19-34, notamment p. 28-34.

9 Voir la critique de P.J. Thomas-Lamotre, Guérir avec Thérèse. Essai sur la maladie intérieure, Paris 2001, p. 94.

10 PA, p. 228. En dépit de cette position, il a prescrit l'hydrothérapie, traitement typique de la névrose hystérique, faute de mieux sûrement.

11 PO, p. 303

12 I.F.G. Durf, L'histoire du fantasme d'une sainte, «Archives de sciences sociales des religions » 41 (1976), p. 117.

13 Voir la bibliographie: K. DABRoWSKI, A. KAWCZAK, M.M. PIECHOWSKI, La croissance mentale par la désintégration positive, Québec 1972 ; K. DĄBROWsKI, La psychonévrose n'est pas une maladie : névroses et psychonévroses, considérées du point de vue de la désintégration positive, Ste-Foy 1972 ; id., Le dynamisme des concepts : phénomènes positifs dans la désintégration, Ste-Foy 1972 ; id., Confé- 
peut se faire que par une désintégration de la structure personnelle de départ, dans une tension qui cause la névrose et le conflit intérieur. Ce processus est positif parce que, somme toute, il conditionne l'apparition d'une personnalité plus mûre. Cette interprétation répondrait mieux au cas de la maladie de Thérèse, qu'elle présenterait non pas tant comme un accident que comme une étape vers la maturité. Père Marie-Eugène de l'Enfant-Jésus, se fondant quant à lui sur la théologie mystique, affirmera aussi le rôle positif de la maladie de Thérèse ${ }^{14}$.

\section{ThÉOLOGIE}

Tel est généralement l'apport de la médecine dans la compréhension de la maladie de Thérèse. Or cette approche ne peut nous satisfaire, du fait de la limitation de son point de vue. Si l'on réduisait l'étude des écrits de Thérèse à une seule lecture psychologique, on n'entendrait pas l'essentiel de son message. Ce n'est pas par goût de l'extraversion qu'elle s'est abondamment épanchée (sept pages écrites) sur cette épreuve (l'analyse graphologique révèle que cette rétrospection lui a beaucoup coûté $^{15}$ ), mais pour communiquer un message de foi. Dans le Manuscrit A le choix des souvenirs a été déterminé par une pensée théologale préalable : «Ce n’est donc pas ma vie proprement dite que je vais écrire, ce sont mes pensées sur les grâces que le Bon Dieu a daigné m'accorder » ${ }^{16}$. Quelle grâce, ou quel « privilège de Jésus sur son âme», veut-elle extraire du souvenir de sa maladie et de sa guérison, et dont ne tient pas compte l'approche psychologique? Pour répondre, voyons d'abord deux approches qui tentent d'expliquer la maladie par des causes différentes : une influence démoniaque et une action de Dieu.

rences. Personnalité, psychonévroses et santé mentale d’après la théorie de la désintégration positive, Warszawa, Paris 1965.

14 Pour aller plus loin, on pourrait imaginer une étude des nuits de saint Jean de la Croix du point de vue de la théorie du professeur Dąbrowski. Nous ne connaissons que trois essais d'interprétation de certaines caractéristiques de la vie spirituelle et mystique à la lumière de la théorie de la désintégration positive : L. NIxON, Meditation as a Means of Actualizing the Religious Personality Ideal : A Review of Empirical Research from the Perspective of Kazimierz Dabrowski's Theory of Positive Disintegration, mémoire de maitrise. Montréal 1983 ; id., The Mystical Struggle : A Psychological Analysis, thèse de doctorat, Montréal 1990 ; C. KozŁowski, Esercizi spirituali di S. Ignazio alla luce della teoria della disintegrazione positiva di K. Dabrowski, thèse de mémoire, Roma 1980.

Dans la note A $30 \mathrm{v}^{\circ}, 11 / 25$ de l'édition Ms NEC nous lisons : «Le graphisme de cette page est très tourmenté : beaucoup de lettres informes, surtout en finale, beaucoup de reprises ; fatigue ou émotion en revivant ces confidences pénibles?". 


\subsection{Action du démon}

Clapier, partant des attestations explicites de Thérèse, soutient que la maladie fut l'effet d'une influence maléfique ${ }^{17}$. L'avis de la carmélite sur ce sujet est effectivement univoque : la maladie vint du démon.

La maladie dont je fus atteinte venait certainement du démon, furieux de votre entrée au Carmel il voulut se venger sur moi du tort que notre famille devait lui faire dans l'avenir ${ }^{18}$.

Je ne sais comment décrire une si étrange maladie, je suis persuadée maintenant qu'elle était l'œuvre du démon ${ }^{19}$.

Je crois que le démon avait reçu un pouvoir extérieur sur moi mais qu'il ne pouvait approcher de mon âme ni de mon esprit, si ce n'est pour m'inspirer des frayeurs très grandes de certaines choses ${ }^{20}$.

Pour Clapier, dont l'intention était de faire concorder les données psychologiques et théologiques, la maladie a eu deux causes : naturelle et extra-humaine maléfique. La cause naturelle étant première (la réalité de la maladie n'est donc pas mise en cause), et la cause diabolique étant seconde, greffée sur le désordre psychologique (ou profitant de lui) :

Une crise à caractère névrotique ou dépressive, traversée d'une influence occulte ${ }^{21}$.

Une maladie psychique engendrant un comportement névrotique ou dépressif traversé d'une influence occulte ${ }^{22}$.

Une influence extra-humaine maléfique $[. .$.$] , capable d'agir négativement sur$ des désordres psychologiques réels, de se greffer, en quelque sorte, sur eux, sans en être pour autant la cause première ${ }^{23}$.

17 Voir J. ClaPIER, «Aimer jusqu'à mourir d'amour ». Thérèse de Lisieux et le mystère pascal, Paris 2003, p. $148-158$.

18 A $27 \mathrm{r}^{\circ}$.

19 A $28 \mathrm{v}^{\circ}$.

20 A $28 \mathrm{v}^{\circ}-29 \mathrm{r}^{\circ}$.

21 J. ClaPier, «Aimer jusqu' à mourir d'amour», p. 158.

22 Ibid., p. 150.

23 Ibid., p. 149. 
Le but de cette influence du démon a été, toujours selon Clapier, d'entraver l'itinéraire de foi de Thérèse ${ }^{24}$.

Sans nous engager à la suite de Clapier sur la place et le rôle de cette influence dans l'ensemble de l'interprétation théologale de la crise, nous pensons qu'il faut respecter le discernement de Thérèse. Et ceci, malgré un certain nombre de contestations, qui semblent relever plutôt du rationalisme que d'une solide argumentation. Ainsi en est-il du jugement selon lequel l'action du démon «ne serait affirmée par Thérèse et ses sœurs qu'en raison d'une ignorance médicale alliée au conditionne-

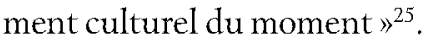

Nous pouvons avancer deux arguments pour soutenir le discernement de Thérèse. Le premier, c'est son autorité comme sainte et docteur de l'Église. Son jugement dans les choses spirituelles devrait compter. Marie-Eugène de l'Enfant-Jésus soutient que les saints parvenus à l'union avec Dieu ont le don de la pénétration spirituelle et du discernement des esprits :

Thérèse l'écrit en son autobiographie au moment où elle est parvenue au sommet de la vie spirituelle. Cette affirmation [de l'influence démoniaque] est donc celle d'un de ces esprits purifiés, auxquels, à la suite de l'apôtre, saint Jean de la Croix et sainte Thérèse d'Avila s'accordent à reconnaitre une pénétration toute particulière des choses de Dieu et des choses d'en-bas, et un discernement, douloureux d'ailleurs, de la présence et de l'action du démon ${ }^{26}$.

Le deuxième argument provient de ce qu'on sait de la stratégie du démon, qui multiplie les obstacles et harcèle ceux qui s'approchent de Dieu, en exerçant son influence par le biais de leurs imperfections. D'après Marie-Eugène de l'Enfant-Jésus, cette stratégie a été mise en œuvre lors de la maladie de Thérèse :

L'enjeu de la rencontre entre le divin et l'humain, entre la pureté de Dieu et l'impureté de l'âme, est trop important pour que le démon n'y intervienne pas de toute la puissance dont il dispose. Encore un peu de temps et l'âme purifiée

24 Voir ibid., p. 151.

25 Voir le résumé de la position d'Albert du Sacré-Cœur par J. Clapier, "Aimer jusqu'à mourir d'amour", p. 150.

26 Marie-EugÈne de l'Enfant-Jésus, La grâce de Noël 1886 chez Ste Thérèse de l'Enfant-Jésus, "Carmel $» 2$ (1959), p. 99. Dans la note on trouve cette citation de Jean de la Croix: «Nous devons savoir que ceux dont l'esprit est complètement purifié peuvent, les uns plus que les autres, avoir la plus grande facilité et comme naturellement connaître ce qu'il y a dans le cœur ou les pensées intimes, les inclinations et les qualités des autres. Ils le connaissent par des indices extérieurs, mêmes très minimes, comme les paroles, les mouvements et autres signes. De même que le démon a ce pouvoir parce qu'il est esprit, de même aussi l'homme spirituel le possède selon cette parole de l'apôtre : Spiritualis autem judicat onnia ; 'l'homme spirituel juge de tout' " (1 Co 2, 15) (La Monté du Carmel, II, 24, traduction du père Grégoire de saint Joseph, Paris 1947¹, p. 268). 
par la nuit de l'esprit sera à l'abri de ses atteintes et lui deviendra terrible. Le démon utilisera les avantages qu'il possède encore sur elle grâce à ses imperfections et à ses attaches au sensible. Saint Jean de la Croix note en effet que : «le démon se poste avec toute sa perfidie sur le passage qui va du sens à l'esprit » (Jean de la Croix, Vive Flamme, III $)^{27}$.

Le démon profite de l'obscurité de ces régions, de l'inexpérience des âmes qui les abordent, de son pouvoir dans le domaine sensible qu'elles n'ont point encore quitté, de sa connaissance merveilleuse des ressorts psychologiques et des tendances de chaque âme, pour augmenter le trouble, la confusion, la souffrance, multiplier les difficultés et renforcer ainsi les obstacles à l'avancement spirituel.

C'est en ce passage du sens à l'esprit que se trouve Thérèse au moment de sa maladie.

À certains indices, le démon avait dû découvrir la valeur de son âme, entrevoir du moins obscurément - sa mission, le mal qu'elle devait lui faire. Nous appuyant sur une affirmation de saint Jean de la Croix, ne pourrait-on pas penser que Dieu lui en avait donné une certaine connaissance. En effet, « Dieu - dit le saint Docteur - permet d'ordinaire que le démon ait connaissance des faveurs qu'Il confère par le moyen du bon Ange, pour qu'il s'y oppose de toutes ses forces d'après les proportions de la justice et ne puisse alléguer de son droit en prétextant qu'on ne lui permet pas de vaincre l'âme, comme il l'a dit de Job » (Nuit obscure, II, 23).

Le démon devait donc normalement mettre en œuvre toutes les ressources de son art ténébreux et perfide autour de Thérèse. Ce serait dépasser les limites habituelles de l'action du démon que de lui attribuer d'avoir provoqué lui-même la maladie ; mais, meneur de jeu intelligent, il a pu et dû utiliser les évènements, dans le cas, les conséquences de la double frustration affective sur le psychisme de sainte Thérèse, pour en augmenter les effets de trouble et de souffrance, retarder le développement normal des facultés de cette enfant et tenter de bloquer ainsi sa marche vers la sainteté. C'est cette action que Thérèse a discernée et qu'elle affirme ${ }^{28}$.

Considérer la maladie de Thérèse comme un effet, même secondaire, du Malin, est un peu réducteur cependant. Selon Clapier, la Sainte aurait eu l'intention de dégager ainsi une vision théologale, dont les principaux artisans sont Dieu appelant et

27 Marie-Eligène de l'Enfant-Jésus, Je veux voir Dieu, Tarascon 1973, p. 761.

28 Ead., La grâce de Noël..., p. 99-100. 
le démon empêchant la réalisation de l'appe ${ }^{29}$. Sur ce point, la proposition de Marie-Eugène de l'Enfant-Jésus va plus loin.

\subsection{La nuit}

Tout en reconnaissant le caractère maladif de la crise de Thérèse ainsi que l'action du démon, l'auteur carmélitain ne s'y s'arrête pas. Il y voit aussi une action de Dieu : «N'est-il pas permis de croire que la maladie étrange que subit sainte Thérèse de l'Enfant-Jésus fut causée à la fois par l'action de Dieu et par l'ébranlement moral produit par l'entrée au Carmel de sa sour Pauline $\star^{30}$.

Comment justifie-t-il sa thèse? En s'appuyant sur l'autorité de saint Jean de la Croix, il rappelle d'abord que le cheminement vers Dieu - et c'est sans doute le cas de Thérèse - se fait nécessairement dans la nuit : «La nuit c'est tout l'itinéraire spirituel de l'âme vers Dieu. Elle est cet itinéraire même $»^{31}$. Et Jean de la Croix de dire : "Pour atteindre l'état de perfection, l'âme d'ordinaire doit auparavant passer par deux nuits principales [...]. Dans l'une et dans l'autre l'âme chemine comme dans l'obscurité de la nuit *32.

Les nuits elles-mêmes peuvent être décrites comme «les privations et purifications par lesquelles l'âme doit passer pour atteindre l'union avec Dieu $»^{33}$. Or, il est important de savoir que, dans la doctrine sanjuaniste, les privations ne concernent pas les biens mais le désir (appétit) de ces biens. C'est le point crucial dans l'enseignement du Docteur mystique :

Nous ne parlons pas ici du manquement effectif, car il ne dénude point l'âme si elle garde le désir de ce qui lui manque. Nous parlons du dénuement de l'appétit et du goût par rapport à tous les biens de ce monde, dénuement qui rend l'âme libre et vide, même en les possédant. En pareil cas, les choses de ce monde n'occupent point l'âme et ne lui nuisent point, parce qu'elles ne pénètrent pas en elle. Ce qui lui nuit, c'est le désir, c'est l'appétit qui subsiste en elle par rapport à ces biens ${ }^{34}$.

Marie-Eugène de l'Enfant-Jésus affirme ensuite que la maladie de Thérèse fut pour elle une pareille nuit du sens : «Cette purification de la sensibilité et du cour,

29 Voir J. ClaPier, "Aimer jusqu'à mourir d'amour ", p. 151

30 Marie-Eugene de L'enfant-Jesus, Je veux voir Dieu, p. 778.

31 Ibid., p. 520.

32 JeAN DE LA Croix, La Montée du Carmel, I, 1, 1, in : id., CEuvres complètes, Paris 1990. Les citations sont données d'après cette traduction. Les cas contraires sont signalés.

33 Marie-Eugene de L'enfant-Jesus, Je veux voir Dieu, p. 520-521.

34 Jean de la Croix, La Montée du Camel, I, 3, 4. 
subie dans son enfance au cours du développement de son affectivité, produit chez Thérèse une blessure : on peut parler certainement d'une 'purification du sens' ${ }^{35}$.

À cette lumière, la purification de Thérèse aurait visé son attachement maladif à la figure maternelle, et le manque de liberté qui s'ensuivait. Ce sera pour elle un problème majeur. Nous la verrons encore au Carmel se cramponner à la rampe de l'escalier pour résister au désir d'aller voir Mère Marie de Gonzague, pour qui elle éprouvait un attrait naturel très fort :

Je me souviens qu'étant postulante, j'avais parfois de si violentes tentations d'entrer chez vous pour me satisfaire, trouver quelques gouttes de joie, que j'étais obligée de passer rapidement devant le dépôt et de me cramponner à la rampe de l'escalier. Il me venait à l'esprit une foule de permissions à demander, enfin, ma Mère bien-aimée, je trouvais mille raisons pour contenter ma nature... ${ }^{36}$.

L'objection qui s'impose naturellement, c'est que la crise de Thérèse apparaît comme une maladie évidente, ce que le Carme ne nie pas, nous l'avons vu. Comment donc y voir une action de Dieu? Nous touchons ici aux limites de la psychologie et de la théologie spirituelle. La psychologie ne peut voir que ce qui relève du phénomène psychologique, et elle ignore la réalité de la foi. Or, pour le théologien, celle-ci doit être prioritaire. En dépit de l'aspect maladif de la crise de Thérèse, on ne peut pas exclure une action de Dieu qui peut s'accompagner, elle aussi, de troubles psychiques, et accentuer (provisoirement) la pathologie. Le texte de saint Jean de la Croix explique comment la purification fait apparaitre les tendances pathologiques :

Le feu matériel, quand il s'attache au bois, commence par le sécher : il en chasse l'humidité et lui fait pleurer l'eau qu'il contient. Il le rend ensuite noir, obscur, désagréable à voir et de mauvaise odeur. Après l'avoir ainsi progressivement séché, il met à nu et chasse dehors tous les accidents obscurs, contraires à la nature du feu $[\ldots]$.

Il en est de même de ce feu d'amour, de cette divine contemplation, par rapport à notre âme. Avant de s'unir cette âme et de la transformer en soi, le feu divin la purifie de tous les accidents contraires qu'il trouve en elle. Il tire au-dehors ses laideurs, il la rend noire et obscure, en sorte qu'elle parait pire qu'auparavant et vraiment laide, abominable. En effet, ces humeurs mauvaises que la divine puri-

35 Marie-Eugène de l'EnFant-Jésus, «Ton amour a grandi avec moi : un génie spirituel, Thérèse de Lisieux, Venasque 1987, p. 36.

${ }^{36} \mathrm{C} 21 \mathrm{v}^{\circ}-22 \mathrm{r}^{\circ}$. 
fication chasse au-dehors étaient si bien fixées, enracinées dans l'âme, qu'elle ne les voyait pas, elle ignorait tout le mal qui était en elle $^{37}$.

Le manque de discernement de l'action de Dieu peut parfois causer des préjudices. Ainsi, le saint polonais Albert Chmielowski (1845-1916) fut mis à la porte d'un noviciat jésuite : alors qu'il traversait la nuit la plus obscure, les supérieurs avaient cru à une maladie psychique. Marie-Eugène de l'Enfant-Jésus pense que l'ébranlement physique de sainte Thérèse d'Avila dans les premières années de sa vie religieuse, et dont elle faillit mourir, fut déclenché par les grâces d'union dont elle était favorisée à cette époque ${ }^{38}$. Écoutons la Réformatrice du Carmel :

Parfois on me crut morte, au point qu'on laissa couler sur mes paupière de la cire que j'y trouvai ensuite. [...] Dans mon monastère, la sépulture était ouverte depuis un jour et demi, attendant mon corps, et un couvent de religieux de notre Ordre, situé en dehors de la ville, avait déjà célébré à mon intention un service funèbre, quand le Seigneur permit que je revienne à moi ${ }^{39}$.

Nous amplifierons la démonstration de Marie-Eugène par notre propre contribution à titre d'hypothèse. Ainsi, en regardant de plus près le Manuscrit A, on constate que souvent les longues confessions de la carmélite ne sont que les développements d'une pensée brièvement énoncée au début. C'est le cas du récit que nous analysons. En effet, juste avant de traiter de la maladie, Thérèse situe l'épreuve dans le mouvement d'une progression spirituelle et non d'une régression : "Il est surprenant de voir combien mon esprit se développa au sein de la souffrance, il se développa à tel point que je ne tardai pas à tomber malade $»^{40}$.

Clapier a éprouvé une difficulté devant ce texte qui lui a paru «énigmatique»:

Qu'entend-elle lorsqu'elle parle du « développement de son esprit » ? Est-ce une maturation accélérée de la pensée, de l'acuité de son intelligence ? Probablement. Quoi qu'il en soit, l'essor de sa personnalité lui parât « surprenant », anormal parce que fondé sur une anxiété qui confine au paroxysme, une angoisse telle que la poussée insolite de «l'esprit » finit par engendrer la maladie : « il se développa à tel point que je ne tardai pas à tomber malade $»^{41}$.

37 JEAN DE LA CROIX, La Nuit obscure, II, 10, 1-2.

38 Voir Marie-Eugène de L'Enfant-Jésus, Je veux voir Dieu, p. 778.

39 Thérèse d'Avila, Livre de la vie, V,9-10, in : ead., Euvres complètes, Paris 1995.

40 A $27 \mathrm{r}^{\circ}$.

41 J. ClAPIER, «Aimer jusqu'à mourir d'amour», p. 148. 
Sa difficulté vient de ce qu'il confond le spirituel et le psychologique. Ce n'est pas l'esprit de Thérèse qui est tombé malade, mais son psychisme. Thérèse l'a précisé avec beaucoup d'acuité : «Je crois que le démon avait reçu un pouvoir extérieur sur moi mais qu'il ne pouvait approcher de mon âme ni de mon esprit $»^{42}$. La carmélite ne serait pas allée jusqu'à confondre le développement de son esprit avec une "maturation de sa pensée ou de l'acuité de son intelligence ", surtout que ce n'est pas le cas chez Thérèse à dix ans. L'un relève du domaine spirituel, les autres du domaine psychique, deux sphères que Thérèse a su parfaitement distinguer. Pendant que l'un se développait, les autres ont défailli.

Cette méprise de Clapier a entraîné une autre : lorsque la carmélite dit que le développement de son esprit a été "surprenant", il pense qu'elle veut dire "anormal ». L'adjectif thérésien n'a pourtant rien de péjoratif ; il signifie l'étonnement de la moniale devant la vraie progression de son esprit. Cette progression a été si grande que la fillette n'a pas tardé à en tomber malade. La maladie a donc résulté de l'inadaptation de Thérèse à l'action grandissante de la grâce, opérant essentiellement au moyen de la souffrance. C'est d'autant plus vraisemblable que l'on sait la place privilégiée que Thérèse attribue à la souffrance comme moyen de sa progression vers la sainteté : "Je ne pensais pas alors qu'il fallait beaucoup souffrir pour arriver à la sainteté, le Bon Dieu ne tarda pas à me le montrer en m'envoyant les épreuves que j'ai racontées plus haut... » ${ }^{43}$.

Les épreuves qu'elle "a racontées plus haut » sont justement l'entrée de Pauline au Carmel et la maladie qui a suivi. Et encore : « je devais passer par le creuset de l'épreuve et souffrir dès mon enfance afin de pourvoir être plus tôt offerte Jésus ${ }^{44}$.

Revenons sur la position de Marie-Eugène de l'Enfant-Jésus afin d'en signaler les difficultés. La première consiste en l'incertitude avec laquelle cet auteur se prononce. Tantôt sa prise de position est hypothétique : "N'est-il pas permis de croire... ", tantôt sûre: "on peut parler certainement d'une 'purification du sens' ». Cette imprécision résulte de la difficulté qu'il a lui-même ressentie à préciser la part respective des différentes causes. Il en est conscient :

42 A $28 \mathrm{v}^{\circ}-29 \mathrm{r}^{\circ}$

$43 \mathrm{~A} 32 \mathrm{r}^{\circ}$. Nous recourons à saint Augustin, à propos du rôle pédagogique de la souffrance : « Notre auteur [Pélage] prétend 'qu'aucun mal n'est la cause d'un bien quelconque'. Comme si le châtiment n'était pas un mal en vertu duquel tant de gens ont, cependant, été amendés ? Il y a donc des maux qui, en vertu de la merveilleuse miséricorde de Dieu, sont salutaires. A-t-il donc subi un bien, le prophète qui a dit: Tu as détourné de moi ton visage et j'ai été bouleversé ? (Ps 29, 8). Assurément non; et, cependant, ce trouble lui fut, en quelque sorte, un remède contre l'orgueil. Car il avait dit au temps de sa prospérité : Rien à jamais ne m’ébranlera (Ps 29, 7), et il s'attribuait à luimême ce qu'il tenait de Dieu. En effet, qu' avait-il qu'il n'ê̂t pas reçu? (1 Co 4,7). C'est pourquoi il avait fallu lui montrer la source de ce qu'il possédait pour que son humilité recouvrât ce que son orgueil avait perdu" (Augustin, La nature et la grâce (De natura et gratia), Paris 1994, p. 295). 
Est-il possible de déterminer la cause de chacun de ces troubles et de faire la part des causes qui interviennent ? [...] Sainte Thérèse de l'Enfant-Jésus souffre à 9 ans ${ }^{45}$ d'un mal étrange dont elle est guérie par le sourire de la Sainte Vierge. Action de Dieu puissante, faiblesse ou déficience de tempérament, action du démon ? Qui pourra soulever le voile du mystère pour faire la part de chacune de ces déterminantes? L'intérêt du problème diminue devant la réussite merveilleuse d'une haute sainteté dans un équilibre humain parfait qui fait de ces âmes des types exceptionnels d'humanité ${ }^{46}$.

Nous pensons, comme lui, qu' il ne faut pas vouloir définir avec trop d'exactitude ce qui relève probablement du second rang. Il est secondaire de connaître les causes ou la nature de la maladie si le but est atteint, à savoir la sainteté incontestable à laquelle Thérèse est parvenue. Le problème de la maladie a déjà été résolu (ou contourné) de la sorte lors du procès de béatification et de canonisation. Le décret de la Congrégation des Rites, reconnaissant l'héroïcité des vertus de sainte Thérèse, ne voulut pas se prononcer sur la nature de la maladie ( « une maladie dont le caractère sembla mystérieux aux médecins $\$^{47}$ ). Il souligna en revanche sa parfaite guérison, condition indispensable pour une maturité psychique et spirituelle: "on doit considérer comme absolument indéniable que, par un prodige de la Sainte Vierge, Mère de Dieu, elle en fut instantanément et parfaitement guérie ${ }^{48}$.

Une deuxième difficulté à signaler, c'est que Thérèse n'interprète pas sa vie selon les catégories de la nuit. Bien qu'on la dise disciple de saint Jean de la Croix, ou un Jean de la Croix du XX siècle ${ }^{49}$, on ne peut la faire rentrer facilement dans le schéma sanjuaniste de la nuit ${ }^{50}$. Comme nous allons le voir, elle a sa propre lecture de la maladie et de la guérison.

45 Thérèse a en fait dix ans.

46 Marie-Eligène de l'Enfant-Jésus, Je veux voir Dieu, p. 808.

47 Congrégation des Rites, Décret sur l'héroïcité des vertus de Sainte Thérèse de l'Enfant-Jésus, AAS 13 (1921), p. 450.

48 Ibid.

49 Voir à ce sujet deux articles: Gregorio De Jesus CruCIFICADo, Las noches sanjuanistas vividas por Santa Teresa del Nino Jesus, EphCarm 2 (1960), p. 352-382. (Traduit en français : Les nuits sanjuanistes vécues par sainte Thérèse de l'Enfant-Jésus, «Supplément de la Vie spirituelle »15 [1962], p. 611-643) ; P. Blanchard, Sainte Thérèse de l'Enfant-Jésus, fille de saint Jean de la Croix, «L'Année Théologique 8 (1947), p. 425-439.

50 Le père von Balthasar écrit que Thérèse, tout en connaissant par cœur les œeuvres de Jean de la Croix, n'a rien adopté du système du saint (voir H.U. von BaLTHASAR, Thérèse de Lisieux - Histoire d'une mission, Paris 1996, p. 58). 


\section{L'EXpérience du SALUT}

On peut réduire à deux éléments qui se répondent le plan du long récit de l'épreuve : en situation de danger - le secours de Dieu. L'un ne va pas sans l'autre. On ne pourra donc parler de la maladie de Thérèse Martin sans évoquer sa guérison, comme dans le récit pascal. En effet, de même que la Passion et la Résurrection du Christ sont un seul mystère, de même la maladie et la guérison de Thérèse sont un seul événement à caractère théologal : l'épreuve devait aboutir à la guérison, et, par là, à la louange de l'œuvre du salut. C'est ainsi qu'elle l'interprète : «cette maladie n'était pas pour que je meure, elle était plutôt comme celle de Lazare afin que Dieu soit glorifié... » ${ }^{51}$.

\subsection{Le danger}

Dans un premier temps, Thérèse parlera de l'abîme de sa faiblesse, pour démontrer ensuite qu'il a attiré l'abîme de la Miséricorde, comme il est dit dans le psaume : «les flots de l'abîme s'appellent l'un l'autre» (Ps 42,8). Fidèle à sa métaphore de la petite fleur blanche du début du Manuscrit $\mathrm{A}^{52}$, elle choisit l'image d'une fleur fragile menacée par la tempête pour rendre compte de sa situation critique : « La douce Reine du Ciel veillait sur sa fragile petite fleur [... ] et s'apprêtait à faire cesser la tempête au moment où sa fleur devait se briser sans retour... " $^{53}$.

Cette métaphore est dramatique : la fleur devait se briser sans retour. Elle exprime toute la menace qui pesait sur la malade, littéralement menace de mort ou, du moins, de folie : le " pauvre petit Père crut que 'sa petite fille allait devenir folle ou bien qu'elle allait mourir' ${ }^{54}$. La situation paraissait sans espoir, arrêtée pour toujours : «la 'petite fleur' languissait et semblait à jamais flétrie " $^{55}$. C'est pour cela que la comparaison avec Lazare lui vient tout naturellement à l'esprit. Comme lui, la fillette devait se considérer engagée sur la même voie de mort, tout le lui disait: la bonne des Guérin reçoit monsieur Martin et ses deux filles aînées rentrés de Paris «avec une figure si triste que Marie crut que j'étais morte... »" ${ }^{56}$ l'oncle Guérin «jugea [l'état] très grave, mais ne voulut pas le dire afin de ne pas effrayer ma Tante ${ }^{57}$. Le médecin dit que c'est une maladie « très grave et dont jamais une enfant si jeune n'avait été atteinte $»^{58}$.

Il y a pire, le Ciel même et la nature paraissent comploter sa perte. Le Ciel, car la maladie se déclare, avec amère ironie, le jour de Pâques, comme s'il se moquait

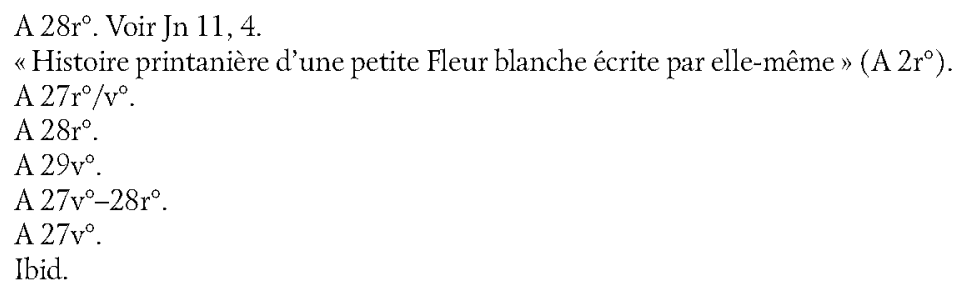


de Thérèse et l'oubliait dans sa victoire sur la mort. On peut y voir l'anticipation de l'autre ironie de Pâques 1896 et de ses interrogations :

Tu rêves la lumière, une patrie embaumée des plus suaves parfums, tu rêves la possession étemelle du Créateur de toutes ces merveilles, tu crois sortir un jour des brouillards qui t'environnent, avance, avance, réjouis-toi de la mort qui te donnera non ce que tu espères, mais une nuit plus profonde encore, la nuit du néant ${ }^{59}$.

Bellet parlera alors de l'inversion des fêtes :

Les choses ne vont pas comme elles devraient ! Rien n'est plus significatif à cet égard que cette inversion de Pâques et du vendredi saint, où le vendredi de la Passion est pour elle jour de joie, le dimanche de la Résurrection entrée dans la nuit ${ }^{60}$.

La nature, elle aussi, semble se rire de Thérèse, parce que « toute la nature se parait de fleurs et respirait la gaîté, seule 'la petite fleur' languissait et semblait à jamais flétrie $»^{61}$.

La situation sans issue est récapitulée dans cette phrase : «la maladie devint si grave que je ne devais pas en guérir suivant les calculs humains... ${ }^{62}$. Toute la description de la maladie a finalement pour seul but d'illustrer jusqu'à quel point sa situation est devenue désespérée. Ainsi Thérèse a-t-elle préparé le lecteur à l'un des plus grands « privilèges » de Jésus pour elle, l'une de ses « prévenances tout à fait gratuites ». Dieu, la tirant de sa détresse, a montré son amour inconditionnel et tendre pour celle qui n'avait plus recours qu'à Lui seul :

«Ne trouvant aucun secours sur la terre, la pauvre petite Thérèse s'était aussi tournée vers sa Mère du Ciel, elle la priait de tout son cour d'avoir enfin pitié d'elle... » ${ }^{63}$.

\subsection{Le secours de Dieu}

La grâce à laquelle devait préparer la maladie a eu son prélude dans l'attitude de l'entourage. L'état d'impuissance où Thérèse s'est vue réduite, lui a donné l'occasion de s'édifier de la résignation de ses proches à la volonté de Dieu, de son père surtout. Dieu a été glorifié par cela d'abord : «Cette maladie était afin que Dieu soit glorifié... Il le fut en effet, par la résignation admirable de mon pauvre petit Père ${ }^{64}$.

$\mathrm{C} 6 \mathrm{v}^{\circ}$

60 M. BelLet, Thérèse et l'illusion, Paris 1998, p. 99.

61 A $29 \mathrm{v}^{\circ}$

62 A $29 \mathrm{r}^{\circ}$

63 A $30 r^{\circ}$.

64 A $28 r^{\circ}$. 
Thérèse admire également sa foi et son amour lorsqu'il fait dire des messes à Notre Dame de Victoires pour la guérison de sa fille ${ }^{65}$.

Elle expérimente aussi l'amour et le dévouement de ses proches. Une grande partie de son récit est consacrée en effet à énumérer ceux qui l'ont soignée ou gardée : Marie a joué le rôle le plus important : "Ah! qu'elle a souffert à cause de moi... combien je lui suis reconnaissante des soins qu'elle m'a prodigués avec tant de désintéressement... $\aleph^{66}$; viennent ensuite l'oncle et la tante, Léonie, Céline et même Pauline qui la soutenait par ses lettres du Carmel. Sachant qu'elle a été une patiente très difficile ( «une pauvre petite fille qui ressemblait à une idiote " ${ }^{67}$ ), Thérèse détaille leurs actes de charité pareils à l'amour tendre que l'on a pour le pauvre : "vraiment il fallait de l'amour pour ne pas me fuir... Ah ! mes chères petites Sœurs, que je vous ai fait souffrir ?... personne ne vous avait fait autant de peine que moi et personne n'avait reçu autant d'amour que vous m'en avez prodigué... $~^{68}$. Cette sincère confession montre qu'elle a accueilli leur dévouement comme un amour immérité et gratuit.

Mais ce n'était que le prélude à la grâce proprement dite. Comme nous l'avons dit, Thérèse a suffisamment démontré l'impasse de sa situation (" ne trouvant aucun secours sur la terre ») pour tourner les regards vers la seule solution possible, un miracle : "Il fallait un miracle et ce fut Notre Dame des Victoires qui le fit " ${ }^{69}$. Ce miracle s'est opéré dans une ambiance de foi profonde : une neuvaine de messes est justement célébrée pour sa guérison à Notre Dame des Victoires de Paris ; Marie, avec ses deux sœurs Léonie et Céline, se met à genoux devant la statue de la Vierge Marie pour l'implorer : « je me jetai avec mes sœurs aux pieds de la Sainte Vierge. Par trois fois, je renouvelai la même prière. A la troisième fois, je vis Thérèse fixer la statue de la Sainte Vierge " ${ }^{70}$. Thérèse même "s'était aussi tournée vers sa Mère du Ciel, elle la priait de tout son cœur d'avoir enfin pitié d'elle... . ${ }^{71}$. Comment ne pas penser au psalmiste priant :

Tire-moi du bourbier, que je n'enfonce, que j'échappe à l'abîme des eaux!

Que le flux des eaux ne me submerge, que le gouffre ne me dévore,

65 Voir A $29 \mathrm{v}^{\circ}$.

66 A $28 \mathrm{r}^{\circ}$.

67 A $29 \mathrm{r}^{\circ}$.

68 A $29 \mathrm{r}^{\circ} / \mathrm{v}^{\circ}$.

69 A $30 \mathrm{r}^{\circ}$.

70 Marie du Sacré-Cœur, PO, p. 241. Notons que c'est grâce à l'instance de Marie que la statue de la Vierge s'est trouvée aux Buissonnets, car le père, au moment du déménagement à Lisieux, craignant les avatars du transport, avait voulu la replacer au Pavillon à Alençon. Sur l'histoire de la statue, voir le livre de S.-J. PIAT, La Vierge du sourire et Sainte Thérèse de l'Enfant-Jésus, Lisieux 1951 (p. 25 sur le détaille qui vient d'être mentionné).

71 A $30 \mathrm{r}^{\circ}$. 
que la bouche de la fosse ne me happe!

Réponds-moi, Yahvé : car ton amour est bonté;

En ta grande tendresse regarde vers moi ;

à ton serviteur ne cache point ta face,

l'angoisse est sur moi, vite, réponds-moi. (Ps 69, 15-18)

Et le miracle tant désiré se produit :

Tout à coup la Sainte Vierge me parut belle, si belle que jamais je n'avais vu rien de si beau, son visage respirait une bonté et une tendresse ineffable, mais ce qui me pénétra jusqu'au fond de l'âme ce fut le «ravissant sourire de la Ste Vierge ». Alors toutes mes peines s'évanouirent, deux grosses larmes jaillirent de mes paupières et coulèrent silencieusement sur mes joues, mais c'était des larmes d'une joie sans mélange... ${ }^{72}$.

Thérèse retient de cette vision la beauté et la bonté du visage de la Vierge. C'est précisément ce qui l'a touchée et a produit la guérison. Pour elle, la beauté de la figure de Marie se confond avec sa bonté et sa tendresse. Elle a fixé son attention uniquement sur ce visage souriant, traduisant au mieux à ses yeux l'amour d'une mère pour son enfant. On s'en rendra compte quelques jours plus tard, à l'occasion du questionnement indiscret des carmélites, qui se sont imaginé dans cette vision « tout autre chose $»^{73}$, que le simple visage souriant de la Vierge :

On me questionna [... ] si la $S^{\text {te }}$ Vierge portait le petit Jésus, ou bien s'il y avait beaucoup de lumière, etc.... Toutes ces questions me troublèrent et me firent de la peine, je ne pouvais dire qu'une chose : «la Sainte Vierge m'avait semblé très belle... et je l'avais vue me sourire ». C'était sa figure seule qui m'avait frappée.

Le visage qu'elle a contemplé est un visage de mère, l'amour qu'elle a ressenti est un amour maternel. Les psychologues ont remarqué à bon escient que la quête de Thérèse durant cette épreuve était une quête filiale. Effectivement, elle a enregistré les événements dans sa mémoire sous cet angle particulier : Marie, écrit-elle, priait la Sainte Vierge «avec la ferveur d'une Mère qui demande la vie de son enfant $»^{74}$; « son cœur lui dictait ce qui m'était nécessaire et vraiment un cœur de Mère est bien plus savant que celui d'un médecin, il sait deviner ce qui convient à la maladie de son enfant... " " $^{75}$ elle «était toujours auprès de mon lit me soignant et me consolant avec

Ibid.

A $31 r^{\circ}$. Ibid. pour les citations suivantes.

A $30 r^{\circ}$.

A $28 r^{\circ}$. 
la tendresse d'une Mère. [...] Je ne souffrais pas qu'elle s'éloigne de moi » ${ }^{76}$; Thérèse l'appelait tout simplement "Mama ${ }^{77}$. De même au sujet de sa tante : elle «me soigna avec une sollicitude vraiment maternelle $\gg^{78}$.

Les médecins font cependant savoir que la guérison n'a pas été complète ; elle le sera la nuit de Noël 1886 :

Thérèse a guéri de sa crise mais non de sa névrose. Par la suite en effet on put voir que le caractère restait inchangé et qu'elle restait aussi bébé, aussi pleurnicheuse, qu' auparavant et jusqu'à recourir encore à la fuite dans la maladie. On notera enfin qu'elle fut atteinte plus tard d'une grave crise de scrupules obsédants. C'est le jour de Noël 1886 que Thérèse changera brusquement et pour toujours ${ }^{79}$.

Nous partageons cette opinion. Non que la vision ait été inefficace : elle n'a pas couronné l'édifice, elle a plutôt contribué à en affermir les fondations (tant ébranlées dans l'enfance de Thérèse) : l'édifice lui-même n'est pas encore visible, il émergera progressivement, avec une phase décisive à Noël 1886, et culminera à l'entrée au Carmel. La moniale le comprend dans ce sens, en recourant de nouveau au symbole de la petite fleur:

La petite fleur allait renaître à la vie, le Rayon lumineux qui l'avait réchauffée ne devait pas arrêter ses bienfaits, il n'agit pas tout d'un coup, mais doucement, suavement, il releva sa fleur et la fortifia de telle sorte que cinq ans après elle s'épanouissait sur la montage fertile du Carmel ${ }^{80}$.

Grâce à la vision, Thérèse a été confirmée dans sa quête filiale. Après un tel miracle, il est compréhensible qu'elle ne veuille pas plus tard renoncer à l'attitude de l'enfant, non pas sur le plan psychologique, qui signifierait l'infantilisme, mais sur le plan spirituel. Quatre mois avant sa mort, rongée par la tuberculose, la moniale gardera toujours en mémoire la grâce du sourire survenue au matin de sa vie, et en demandera une autre, par quoi elle reconnaîtra la pleine légitimité et efficacité de la première grâce :

Toi qui vins me sourire au matin de ma vie

Viens me sourire encor... Mère... voici le soir !... ${ }^{81}$

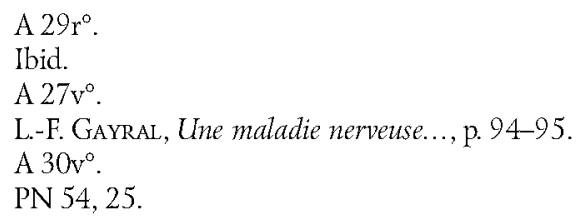


La Vierge Marie est devenue désormais sa Mère par excellence. Thérèse aura une grande piété mariale, et ne voudra pas mourir sans lui consacrer le dernier poème de sa vie (PN 54, «Pourquoi je t'aime, ô Marie!»). Marie a restitué l'image de la mère, assombrie puis disparue précocement. Thérèse a retrouvé dans le visage de la Vierge la douceur de la mère. C'est pourquoi Marie sera pour elle plus Mère que Reine.

\section{BIBLIOGRAPHIE}

Balthasar von H.U., Actualité de Lisieux, « Nouvelles de l'Institut Catholique de Paris » 2 ( $\mathrm{n}^{\circ}$ spécial, mai 1973).

Balthasar von H.U., Thérèse de Lisieux - Histoire d'une mission, Paris 1996.

Bellet M., Thérèse et l'illusion, Paris 1998.

Clapier J., "Aimer jusqu'à mourir d'amour ". Thérèse de Lisieux et le mystère pascal, Paris 2003.

Jean de la Croix, CEuvres completes, Paris 1990.

Marie-Eugène de L'Enfant-JÉsus, "Ton amour a grandi avec moi » : un génie spirituel, Thérèse de Lisieux, Venasque 1987.

Marie-Eugène de l'Enfant-JÉsus, Je veux voir Dieu, Tarascon 1973.

Masson R., Souffrance des hommes. Un psychiatre interroge Thérèse de Lisieux, Versailles 1997.

Meester De C., Dynamique de la confiance. Genèse et structure de la "voie d'enfance spirituelle" de sainte Thérèse de Lisieux, Paris $1995^{2}$ (éd. révisée).

Procès de béatification et canonisation de sainte Thérèse de l'Enfant-Jésus et de la Sainte-Face. I Procès informatif ordinaire, coll. «Bibliotheca carmelitica ", Series I : textus, n², Roma 1973.

Procès de béatification et canonisation de sainte Thérèse de l'Enfant-Jésus et de la Sainte-Face. II Procès apostolique, coll. "Bibliotheca carmelitica ", Series I : textus, $\mathrm{n}^{\circ}$ 3, Roma 1976.

ThérÈse D’Avila,CEuvres complètes, Paris 1995.

Thérèse De L'EnFANT-JÉsus et DE LA SAINTE-FACE, Manuscrits autobiographiques. Nouvelle édition du Centenaire, Édition critique des oeuvres complètes de Sainte Thérèse de l'Enfant-Jésus et de la Sainte-Face, Paris 1992.

Thomas-Lamotte P.J., Guérir avec Thérèse. Essai sur la maladie intérieure, Paris 2001. 


\author{
ABSTRACT \\ Krzysztof PAWEowski OCD \\ The Sickness of Therese Martin and Her Healing \\ by the Smile of the Virgin Mary
}

The sickness from which Therese was suffering when she was ten years old was diagnosed by physicians as neurosis. She diagnosed it herself as an effect of a demon's attack. Are these two different opinions so contradictory that they exclude each other? No necessarily so. John Paul II, in his Fides et ratio, says that truth acquired through natural reason does not render useless the truth of faith. While appreciating the medical point of view, a theologian should not be satisfied with this level of interpretation. It is so in this case because Therese dedicated seven pages of her autobiography to recounting her difficult experience in order to express, above all, her faith.

"The sickness came from the demon." We should respect this as Therese's discernment. She expressed her opinion at the time she was writing The Story of a Soul. This means that it came about when she already reached the summit of union with God. In this period of spiritual life, a person receives the gifts of spiritual perception and the discernment of spirits. According to Saint John of the Cross, Satan driven by jealousy becomes very active toward a person who, progressing on his or her way to God, is at the moment of transition from sensual tasting to the solitude of the spirit. It does not rule out the activity of God, who while introducing the soul of Therese to the night of the senses, wants to set Therese free from disordered attachments to the image of her mother, however she might be depicted in the mind of a ten-year-old child.

Therese does not analyze her life in terms of St John of the Cross' "dark night" but has her own interpretation of events. If the Passion and Resurrection of Christ are one paschal mystery, in the same way, sickness and the return to good health are one theological event. A situation that looked hopeless from a human point of view had to lead to a surprising healing, and through it, to amazement because of this act of salvation. "The sickness was not meant to lead me to death but is more like a sickness of Lazarus, which endowed God with glory."

Słowa klucze: Teresa z Lisieux, Teresa od Dzieciątka Jezus, Teresa Martin, Jan od Krzyża, noc, choroba, nerwica, uzdrowienie, uśmiech Najświętszej Maryi Panny

Keywords: Therese of Lisieux, Therese of the Child Jesus, Therese Martin, John of the Cross, night, sickness, neurosis, healing, the smile of the Virgin Mary 